\title{
Test Result Report
}

National Cancer Institute

\section{Source}

National Cancer Institute. Test Result Report. NCI Thesaurus. Code C70950.

An account of the outcome of an analysis or test. 